\title{
Formación de ciudadanía y diálogo intercultural en el Plan Nacional de Educación Intercultural Bilingüe
}

\author{
Training for citizenship and intercultural dialogue in the \\ National Cross-Cultural Bilingual Education Plan
}

Julio Carrascal Pretell $\unrhd$

Universidad Nacional Federico Villarreal. Lima, Perú

\begin{tabular}{llll}
\hline Recibido: 17/09/2019 & Revisado: 22/11/2019 & Aceptado: 19/02/2020 & Publicado: 18/06/2020
\end{tabular}

\section{RESUMEN}

Este trabajo indaga sobre el documento principal del Plan Nacional de Educación Intercultural Bilingüe (PNEIB) al 2021 en el Perú. Esa propuesta educativa pone de relieve la revaloración de las lenguas originarias, la formación de ciudadanía y el diálogo intercultural entre los diversos actores sociales vinculados al proyecto. Entonces, esta investigación estudia cada uno de los tres ejes anteriores para dar cuenta de su eficaz implementación a partir del análisis discursivo del programa. Para ello, se emplea la propuesta de la interculturalidad crítica, el enfoque de las capacidades y la perspectiva del desarrollo del capital humano. Entonces, se analiza si el proyecto formulado por el PNEIB solo se sostiene a nivel discursivo o tiene un correlato efectivo en la implementación de esta política educativa.

Palabras clave: Educación intercultural bilingüe, interculturalidad, lenguas originarias, ciudadanía, diálogo intercultural.

\begin{abstract}
This work looks into the main document of the National Cross-Cultural Bilingual Education Plan (PNEIB in Spanish) 2021 in Peru. This educational proposal highlights the revaluation of native languages, citizenship training and cross-cultural dialogue among the various social agents linked to the project. Thus, this research studies each of the above three issues to account for their effective implementation based on the discourse analysis of the program. For this purpose, the critical inter-culturality proposal, the abilities approach and the human capital development perspective are used. We then analyze if the project formulated by the PNEIB is viable only at discourse level or has an effective correlation in the implementation of this education policy.
\end{abstract}

Keywords: Intercultural bilingual education, inter-culturality, native languages, citizenship, crosscultural dialogue. 


\section{INTRODUCCIÓN}

El Ministerio de Cultura del Perú reconoce la existencia de 55 pueblos indígenas que utilizan 47 lenguas originarias dentro del territorio nacional (BDPI, 2016). Este panorama ha generado, desde hace algunas décadas, un interés constante del Estado sobre la situación cultural de la nación y sus implicancias en el ámbito educativo. La heterogeneidad cultural del país ha supuesto una complejidad de base para la implementación de las políticas educativas estatales. Sin embargo, si bien la preocupación gubernamental en torno a este eje ha sido exhibida, solo se ha producido a nivel discursivo, pues únicamente se han implementado planes con muchas falencias y que no incorporan real, correcta y oportunamente a las culturas indígenas. A pesar de ello, en los últimos años, se han generado cambios y se ha intentado insertar el enfoque intercultural a las propuestas educativas (Tubino, 2005).

Es ante este escenario que la selección de la política educativa a desarrollar y analizar en el presente trabajo será el Plan Nacional de Educación Intercultural Bilingüe al 2021 (PLANEIB). Este plan es una herramienta de gestión elaborada y construida en un proceso que comprendió cuatro fases, entre las cuales se halla la convocatoria y consulta previa entre el año 2015 y 2016 a especialistas y expertos académicos de ONG's peruanas e internacionales, líderes de comunidades indígenas andinas y amazónicas, maestros y maestras bilingües. También, se han desarrollado talleres macroregionales para discutir la situación y diagnóstico situacional de la educación Intercultural Bilingüe (EIB). Asimismo, incluyó una consulta técnica con la participación de la Comisión Nacional de Educación Intercultural y Bilingüe (CONEIB) que permitiera el armado de una matriz de planeamiento. Finalmente, se implementó la articulación de la revisión del diagnóstico con la planificación de estrategias y actividades del PLANEIB con proyección a que se le dé cumplimiento con metas específicas al 2021.

El Plan Nacional de Educación Intercultural Bilingüe al 2021 tiene entre sus referentes normativos la Declaración de las Naciones Unidas (2007) y la Constitución Política del Perú (1993) respecto de los derechos de los pueblos indígenas. A partir de estos, se construyen las acciones, soportes y objetivos referidos al derecho a una educación de acuerdo con la cultura de origen, lengua materna y herencia cultural.

Los alcances de este plan contemplan cuatro ejes para su implementación, que incluyen el acceso, permanencia y culminación de la educación intercultural bilingüe (EIB), la aplicación del currículo y propuesta pedagógica EIB (con materiales educativos pertinentes a los estudiantes), la formación docente y la gestión descentralizadaparticipativa que atiendan a las demandas educativas en EIB.

El plan nacional de EIB sirve como sustento a la política general educativa de la nación. La EIB se enfoca en el desarrollo educativo de las poblaciones originarias y que habitan en territorios alejados de la urbe capitalina. Asimismo, se interesa por los grupos de individuos que tengan como lengua materna a un dialecto indígena, pero que se desenvuelven en ámbitos urbanos. En otros términos, esta propuesta busca velar por la atención de los aprendizajes de un sector importante de la población nacional y que, tradicionalmente, ha sido relegada de este derecho fundamental. Por tanto, revisar y analizar críticamente este planteamiento servirá para conocer los avances en la 
inclusión equitativa de las culturas indígenas en la construcción de un proyecto nacional. Además, posibilitará generar un mayor conocimiento y aporte sobre los límites de esta postura y mejorarla para que se convierta en un mecanismo importante de formación de ciudadanía. Asimismo, permitirá focalizar la atención para canalizar el empoderamiento social de estas poblaciones vulnerables.

Se han planteado tres preguntas a través de las cuales se orienta en análisis crítico de la política educativa seleccionada. Así, esta elaboración ha sido construida bajo el enfoque de capital humano, el enfoque de capacidades y el enfoque intercultural, las cuales problematizan los alcances de la EIB según las siguientes interrogantes:

¿En qué medida el PNEIB permite la revaloración de las lenguas originarias?

¿El PNEIB promueve la formación de ciudadanía y la agencia en las comunidades indígenas?

¿El PNEIB promueve un diálogo intercultural eficaz?

\section{Revaloración de las lenguas originarias}

El análisis que realiza Catherine Walsh en el año 2009, acerca de la interculturalidad ha formulado tres instancias o modos en que se presenta en el entorno latinoamericano. En primer lugar, propone la existencia de una interculturalidad relacionar que solo se enfoca en el mero contacto cultural; es decir, en un vínculo casi ascético, ya que no toma en cuenta los contextos de la interacción cultural. En otros términos, "hace referencia de forma más básica y general al contacto e intercambio entre culturas" (Walsh, 2009). En segundo lugar, aparece la interculturalidad funcional. Esta se refiere a la diversidad cultural, pero sin cuestionar las asimetrías, desigualdades e inequidades. En otras palabras, tiene su sustento "... en el reconocimiento de la diversidad y diferencias culturales, con metas a la inclusión de esta al interior de la estructura social establecida." (Walsh, 2009). Esta perspectiva sirve como herramienta para la inclusión de una cultura marginada en la matriz dominante. Al no denunciar la falta de equidad, avala una apropiación cultural de la metrópoli sobre las comunidades originarias. En tercer lugar, se presenta la interculturalidad crítica que sí pone en relieve la matriz de poder-étnico-racial. Entonces, se considera que "... reconocimiento de que la diferencia se construye dentro de una estructura y matriz colonial de poder radicalizado y jerarquizado" (Walsh, 2009). Esta postura crítica pone énfasis en el señalamiento de las causas de la dominación; por tanto, estas deben ser mencionadas y se debe evidenciar cómo los procesos están más allá de los discursos. A pesar de nacer y responder al contexto particular de Ecuador, para los fines del análisis, tomaremos como referentes a los dos últimos aspectos de los planteamientos de Walsh para luego de exponer los planes de la EIB.

En los planteamientos generales de la EIB, se señala que esta "busca garantizar el derecho que tiene toda persona a una educación de acuerdo a su cultura y en su lengua materna cuando esta es una lengua originaria, pero también el derecho de los estudiantes de pueblos originarios y/o indígenas a revitalizar su lengua de herencia... asegurándoles a su vez el acceso a otros escenarios socioculturales, al aprendizaje del castellano como lengua para la comunicación nacional, y al de una lengua extranjera para la comunicación en contextos más amplios" (Ministerio de Educación, 2016). Estos lineamientos hacen hincapié en la pericia y del aprendizaje del español como mecanismo para vincularse 
con una cultura ajena a lo indígena. Si bien se menciona el derecho a la enseñanza en alguna lengua aborigen, la revitalización lingüística se supedita a la asimilación del castellano y de una tercera lengua.

La política propuesta por el PNEIB pretende revalorar las lenguas originarias. Por ello, incluye y fomenta su uso dentro de la escuela a partir de las particularidades de cada comunidad. Sin embargo, otorga predominio "al aprendizaje del castellano como lengua para la comunicación nacional, y al de una lengua extranjera para la comunicación en contextos más amplios" (Ministerio de Educación, 2016). En este sentido, usar una lengua indígena solo es funcional para el desarrollo de capacidades y para que los miembros de las comunidades indígenas "accedan a otros códigos culturales de uso nacional e internacional" (Ministerio de Educación, 2016). Por tanto, es posible inferir que la EIB "tiene aún un marcado sesgo lingüístico" (Tubino, 2015) y no fomenta la inclusión del aprendizaje de otros horizontes culturales distintos a lo occidental hegemónico. Este planteamiento solo se orienta a un solo componente cultural, la lengua, y obvia a otros elementos.

Por otro lado, el PNEIB solo responde a una interculturalidad funcional, ya que sirve como mecanismo para la inclusión de las comunidades originarias en la matriz occidental a través del discurso de la posibilidad de inserción en el mercado laboral. Este busca producir réditos económicos que no solo beneficien a los individuos, sino que, también genere repercusiones en las cuentas nacionales. Desde el enfoque del desarrollo del capital humano, se menciona:
"Investment in education has risen at a rapid rate and by itself may well account for a substantial part of the otherwise unexplained rise in earnings. I shall do no more than summarize some preliminary results about the total costs of education including foregone by students, the apparent relation of these costs to consumer income and to alternative investments, the rise of stock of education in the labor force, returns to education, and the contribution that the increase in the stock of education may have made to earnings and to national income" (Schultz, 1961).

Esta postura sostiene que la educación puede ser una herramienta eficaz de desarrollo, aunque se valora, únicamente, la producción monetaria y que sea una forma de reinversión. Según Schultz y sus seguidores, el sistema educativo es el factor que puede combatir las desigualdades, porque incrementa la capacidad productiva y contribuye al incremento de los ingresos.

Según la teoría del capital humano, se ofertaría igualdad de oportunidades y movilidad social gracias a que la inserción en el sistema educativo permite desarrollar habilidades y destrezas y, a través de la meritocracia, las personas podrían obtener un empleo de alta remuneración. Sin embargo, este planteamiento no considera que los individuos parten de situaciones y condiciones diferentes, lo que imposibilita un desarrollo eficiente. Por tanto, el PNEIB y su idea de interculturalidad se sustentan en una perspectiva funcional para el desarrollo del capital humano, puesto que al no cuestionar las inequidades socioeconómicas que padecen los pueblos indígenas, solo buscan generar beneficios a las cuentas macroeconómicas del país. No tomar en cuenta los horizontes culturales 
relegados y pretender tener pericia solo en el uso del castellano produce sujetos que no tienen la agencia necesaria para revitalizar y empoderar su cultura de origen.

En síntesis, el PNEIB no promueve la revaloración de las lenguas originarias, dado que responde, únicamente, a la interculturalidad funcional, sirve solo al desarrollo del capital humano como retorno económico y no implementa la interculturalidad crítica para cuestionar las asimetrías. Entonces, "el discurso sobre la interculturalidad y la educación bilingüe que se imparte desde el Estado. Es un discurso culturalista acrítico sin contenido político y socioeconómico." (Tubino, 2015). Desde el Estado y la política educativa analiza$\mathrm{da}$, se presupone al lenguaje dominante, el español, como la lengua oficial y de las mayorías, lo que implica la asimilación de minorías locales a lo mayoritario y hegemónico. Por tanto, es posible argumentar que el EIB no fomenta la valoración positiva de las lenguas indígenas, pues su uso educativo es solo mecánico y serviría solo dentro de las aulas. Su utilización permanece aislada dentro de las comunidades y las horas de clase impartidas en ellas. En otros términos, "hay firmes evidencias que los lenguajes no pueden sobrevivir por mucho tiempo en el mundo moderno a no ser que sean usados en la vida pública, de esta manera, las decisiones gubernamentales... son decisiones acerca de qué lenguas prosperarán y cuáles se extinguirán" (Kymlicka, 2001). La utilización marginal de las lenguas aborígenes también se vincula por su nula o poca valoración social, puesto que no se ha creado formas de prestigiarlas eficazmente. Es decir, "El uso del lenguaje no puede asumirse como algo meramente técnico, en el sentido de algo objetivo y racional que simplemente es, sino que siempre está atrave- sado por el prestigio, el estatus y la valoración" (Zavala, 2007).

\section{Formación de ciudadanía}

El enfoque del desarrollo de las capacidades propuesto por Amartya Sen (2000) realiza una crítica a la perspectiva del desarrollo del capital humano que iguala el desarrollo con la riqueza económica. El enfoque de las capacidades niega que desarrollarse solo implica el aumento de ingresos monetarios y que el fin no es este. El incremento de réditos solo es un medio para un fin mayor que es la libertad de los individuos. Sin embargo, esta libertad se encuentra limitada por algunos factores de conversión como la pobreza o la desigualdad; por lo que se puede argüir que el individuo construirá su libertad por medio de negociaciones con factores externos a él.

Desde la política educativa estudiada, se propone "Garantizar aprendizajes pertinentes y de calidad a los niños, niñas, adolescentes, jóvenes, personas adultas y personas adultas mayores de todo el Perú, pertenecientes a los pueblos indígenas $u$ originarios que contribuyan a la formación de ciudadanos y ciudadanas protagonistas y en la construcción de un proyecto colectivo de sociedad..." (Ministerio de Educación, 2016). Es decir, se intenta asegurar la calidad educativa de todas las comunidades y grupos etarios del país. Sin embargo, este proyecto excede la capacidad del mismo MINEDU, pues pretende educar incluso fuera de las aulas; por ejemplo, se menciona la educación de adultos mayores que no forman parte del sistema educativo y de escolarización. Asimismo, fomenta el protagonismo ciudadano, pero sin definir la ciudadanía que se desea conseguir.

Si bien, a nivel discursivo, el EIB impulsa la formación de ciudadanos y, por ende, el desarrollo de sus libertades y capacidades, 
aún se fomenta solo la libertad económica. Esta última se entiende como el simple aumento de recursos económicos, ya que el enfoque del PNEIB no cuestiona los factores de conversión presentes en las poblaciones indígenas como la desigualdad social. La presunta difusión y mejoramiento de las capacidades solo es instrumental a una economía que busca rentabilidad, puesto que la ciudadanía requiere de agencia y la libertad para desarrollarla. Sin embargo, no se toma en consideración los factores externos al individuo para la formación de su agencia y, por ende, el aumento de su protagonismo ciudadano. En el EIB, aún se pondera, únicamente, la libertad como capacidad para la inversión económica y no se incluyen otros aspectos relevantes para la construcción efectiva de ciudadanía como el prestigio social de las lenguas originarias.

El PNEIB tiene como finalidad contribuir a la formación y construcción de ciudadanos y ciudadanas de los pueblos indígenas a los que va dirigido el programa. Para comprender y analizar los mecanismos que promueve para ello, es necesario revisar la interculturalidad vista desde la perspectiva del Estado, en contraste con los discursos emitidos desde los movimientos indígenas y pueblos originarios. Ese es un punto de discusión que hace falta analizar, ya que se parten de supuestos diferentes. "En este contexto se entiende como la revalorización y el fortalecimiento de las identidades étnicas... implica la defensa de los territorios ancestrales y la educación bilingüe..." (Tubino: 2015), con lo cual se hace referencia a un tipo de interculturalidad que se refuerza con el derecho al territorio y a la identidad política desde lo que conforma lo étnico. Ello es contrastado con el discurso "oficial" que no define claramente los límites que lo enmarcan. La construcción de la ciudadanía involucra, desde diferentes niveles de participación social, el ejercicio de los derechos básicos de los pueblos. A partir de la realidad asimétrica que se vive en el país, hace falta un modelo renovado de ciudadanía que incorpore y proyecte diversos horizontes culturales, donde lo intercultural deje de ser solo un discurso teórico. Entonces, "ser ciudadano intercultural es ejercer el derecho a construirse una identidad cultural propia y no limitarse a reproducir en uno mismo ni la identidad heredada ni la identidad que la sociedad mayor nos fuerza a adoptar por todos los medios" (Tubino, 2015).

El PNEIB no promueve eficazmente la formación de ciudadanía y la capacidad de agencia de las comunidades originarias. Por un lado, no define lo que es ciudadanía y menos aún la ciudadanía que se espera crear dentro de un proyecto de nación que emana del Estado. Consolidar la formación ciudadana es indispensable para que los sectores marginados consigan agencia necesaria para su desarrollo. Ciudadanía y agencia son indesligables. Por otro lado, "la capacidad de agencia es una propiedad combinada, por eso supone no solo el crecimiento de impulsos internos sino también de condicionamientos externos sin los cuales no es posible la ciudadanía como praxis del desarrollo" (Tubino, 2015). Es decir, se requiere que el EIB incida en el análisis y mejora de las condiciones que posibilitan el desarrollo educativo. Por tanto, una eficaz implementación de la política intercultural bilingüe supone crear un entorno adecuado para su progreso. El PNEIB se desarrolla casi exclusivamente a nivel declarativo, pues únicamente, hace énfasis en un solo aspecto de su propuesta. Si bien el incremento y avances en el acceso son importantes e innegables, solamente se postula la producción de un plan curricular adecuado a los contextos de cada población, la mejora de las condiciones laborales de los maestros 
que son parte del proyecto, la potenciación de la gestión descentralizada y el incremento de la participación social. Estos elementos son mencionados, pero no son conceptualizados, ni se propone un plan para su ejecución.

\section{Diálogo intercultural}

El diálogo intercultural es una postura que fomenta la interacción, intercambio, conocimiento y comprensión entre diversas culturas. Para consolidar esta perspectiva, no deberá imponerse condiciones al diálogo, sino que su desarrollo debe construirse en constante interrelación horizontal entre los diferentes mundos y entornos culturales. Además, se tendrá en cuenta las condiciones en las que se realizará el diálogo como elemento fundamental del mismo. También, "Se trata de un proceso histórico - con sus cursos y sus recursos - que involucra la creación progresiva de nuevos estilos de convivencia, más flexibles y permeables; y de nuevas formas de entender y poner en práctica el ejercicio diferenciado de la ciudadanía" (Tubino, 2016). Asimismo, se requiere reconocer las diferencias, tanto en el ámbito privado como público, para evitar la verticalidad de las relaciones y la homogeneización cultural.

En el 2016, la política educativa intercultural bilingüe menciona que "es fundamental diseñar e implementar el Modelo de Servicio EIB... En relación con ello, es necesario mejorar el desarrollo de programas especializados de enseñanza del castellano como segunda lengua (para la forma de atención respectiva), el tratamiento del enfoque intercultural y el diálogo de saberes que permita articular los conocimientos (Ministerio de Educación, 2016). Se establece la implementación de la interculturalidad y la necesidad de diálogo. Por un lado, como se mencionó, la perspectiva intercultural es un intento fallido de valoración positiva de las lenguas y conocimientos indígenas. Por otro lado, el reconocimiento de los saberes de los pueblos originarios no tiene una formulación específica para su ejecución y la valía de la ciencia aún permanece por encima del conocimiento indígena; es decir, todavía se mantiene relegado del prestigio social.

Respecto a los modelos de intervención del PNEIB, se mencionan tres formas de acción. En primer lugar, aparece la EIB de fortalecimiento lingüístico que está "dirigida a atender de manera pertinente a estudiantes de pueblos originarios y/o indígenas que tienen la lengua originaria como lengua materna y que deben aprender el castellano como segunda lengua" (Ministerio de Educación, 2016). En segundo lugar, se presenta la EIB de revitalización cultural y lingüística. En tercer lugar, se menciona la EIB en ámbitos urbanos. Para estas dos últimas, la política educativa dice que "Esta forma de atención opera por demanda explícita de la comunidad y la escuela" (Ministerio de Educación, 2016). Entonces, se puede entender que se formulan dos mecanismos de atención. Primero, para fortalecer una lengua, el Estado brinda atención educativa en la lengua materna para el aprendizaje del español. Entonces, el fortalecimiento se supeditada al conocimiento de una segunda lengua. La revaloración lingüística se configura como una herramienta para que las comunidades indígenas internalicen una cultura diferente a la suya. Se destaca que este mecanismo es normativo, pues se pondera el 'deber' aprender del castellano y no se especifica la forma de fortalecer, efectivamente, las lenguas originarias. Segundo, la revitalización de la lengua y la atención urbana de la EIB no emana del Estado como una política nacional incluyente. Implementar la EIB solo 
es posible, si alguna comunidad hace un pedido al gobierno. Este modo de tratamiento evidencia el desinterés estatal, ya que la práctica intercultural no es prioritaria al no ser obligatoria en aquellos contextos.

La PNEIB no promueve un diálogo intercultural, pues la propuesta del EIB no cuestiona las condiciones de su implementación y deja de lado el peso sociohistórico que enmarcan las relaciones culturales, las que podrían limitar el desarrollo adecuado de las comunidades indígenas y sus lenguas. Asimismo, el supuesto de la interacción de lenguas en equidad no se cumple, ya que las modalidades de atención tienen un carácter excluyente. Respecto al ámbito rural, la EIB se focaliza en su ejecución dentro de la Educación Básica Regular (EBR) y, en particular, en la educación inicial y primaria. Enfocarse en solo dos niveles educativos demuestra la discontinuidad del programa. La posible revaloración cultural y lingüística lograda en esos primeros años de educación se verá mermada debido al recorte de su implementación. Si bien se enfatiza el aprendizaje intercultural en los niños y adolescentes, no se continua con el plan de revalorización y de producción de prestigio social al excluir la educación secundaria y el sistema universitario. Respecto al ámbito urbano, el PNEIB no ofrece una propuesta de atención educativa con enfoque intercultural. Únicamente, se menciona que la EIB se hará presente por demanda expresa de la comunidad. Es decir, ni la EBR ni la educación universitaria tienen la obligación de incluir esta perspectiva en su sistema de enseñanza.

\section{CONCLUSIONES}

EI PNEIB desarrolla un discurso meramente declarativo de intenciones sobre la interculturalidad y sus implicancias para la revaloración cultural y lingüística de los pueblos originarios del Perú. Se incluyen una serie de normas y principios que, no tienen repercusión en la realidad y siguen siendo solo enunciaciones que no permiten la agencia de las comunidades marginadas.

La propuesta del PNEIB no incluye la educación secundaria y universitaria. Incluso el propio carácter constitutivo de la EIB no le permitirá influir en la educación de adultos mayores.

La política educativa del PNEIB evade el cuestionamiento del origen de las inequidades y desigualdades. Asimismo, se interpreta el ámbito sociocultural de la nación desde un paradigma monocultural que solo valora la perspectiva dominante, la cual busca la asimilación o la homogeneización cultural de los pueblos indígenas.

Las acciones y actividades propuestas por el PNEIB generan discontinuidad en la propuesta general de revaloración.

Las propuestas del PNEIB del 2016 no son eficaces ni efectivas para la revaloración lingüístico-cultural, la formación de ciudadanía y capacidad de agencia y la implementación de un dialogo intercultural.

\section{REVISIÓN BIBLIOGRÁFICA}

Defensoría del Pueblo. (2016). Educación Intercultural Bilingüe hacia el 2021.Una política de Estado imprescindible para el desarrollo de los pueblos indígenas. Serie de Informes Defensoriales: Informe N 174. Lima: Defensoría del Pueblo.

Kymlicka, W. (2001). Politics in the vernacular:Nationalism, Multiculturalism, and Citizenship.Chapter: Human Rights and Ethnocultural justice. Nueva York: Oxford.

Ministerio de Educación. (2016). Plan Nacional de Educación Intercultural Bilingüe al 2021. Lima: Minedu. 
Resolución Ministerial N³36-2016-MC http:// bdpi.cultura.gob.pe/lista-de-pueblos-indígenas

Rodríguez, M. (2018). Construir la interculturalidad. Políticas educativas, diversidad cultural y desigualdad en Ecuador. En: Íconos. Revista de Ciencias Sociales. Núm. 60, enero 2018, pp. 217-236. Ecuador: Facultad Latinoamericana de Ciencias Sociales.

Schultz, T. (1961). Inversión en capital humano. The American Economic Review Vol. 51, $N \circ$ 1. págs. 1-17. https://www.jstor.org/stable/1818907

Sen, A. (2000). Desarrollo y Libertad. Cap. 2 Los fines y los medios del desarrollo.p.54-75. Capítulo 4 "La pobreza como privación de capacidades". p. 114-141. Buenos Aires: Planeta.

Tubino, F. (2008). No una sino muchas ciudadanías: una reflexión desde el Perú y América Latina. En: Cuadernos Interculturales, vol. 6, núm. 10, primer semestre, p. 170-180. Universidad de Playa Ancha. Viña del Mar, Chile.

Tubino, F. (2015). La interculturalidad en cuestión. Lima: Fondo editorial PUCP.

Tubino, F. (2016) La interculturalidad en cuestión. Fondo Editorial, Pontificia Universidad Católica del Perú, 2015.

Tubino, F. y Zariquiey, R. (2005). Las prácticas discursivas sobre la interculturalidad en el Perú de hoy. Propuesta de lineamientos para su tratamiento en el sistema educativo peruano. Consultoría encargada por la Dirección Nacional de Educación Bilingüe Intercultural.

Vaughan, R. (2015). Education, Social Justice and School Diversity: Insights from the Capability Approach. En: Journal of Human Deve- lopment and Capabilities. Vol. 17, No. 2, 206 -224. DOI: 10.1080/19452829.2015.1076775

Walsh, C. (2009). Interculturalidad, Estado, Sociedad: Luchas (de) coloniales de nuestra época. Quito: Universidad Andina Simón Bolívar/Abya Yala.

Zavala, V. (2007). Deconstruyendo la educación intercultural bilingüe: los aportes de la sociolingüística crítica. En: Crónicas urbanas: Análisis y perspectivas urbano-regionales, Año 13, no. 14 Lima. 